\title{
A descriptive study highlighting the differences in the treatment protocol for oral tongue cancer in Sweden and Finland
}

\section{Mäkitie, Antti}

2020-02-01

Mäkitie , A , Kamali , A, Mroueh, R , Lindford , A, Koivunen , P , Autio , T , Lassus , P , Halle , M , Bäck , L , Palmgren , B \& Hammarstedt-Nordenvall , L 2020 , ' A descriptive study highlighting the differences in the treatment protocol for oral tongue cancer in Sweden and Finland ' , Acta Oto-Laryngologica, vol. 140 , no. 2 , pp. 188-194 . https://doi.org/10.1080/00016489.2019.1699663

http://hdl.handle.net/10138/323316

https://doi.org/10.1080/00016489.2019.1699663

unspecified

acceptedVersion

Downloaded from Helda, University of Helsinki institutional repository.

This is an electronic reprint of the original article.

This reprint may differ from the original in pagination and typographic detail.

Please cite the original version. 


\section{A descriptive study highlighting the differences in the treatment protocol for oral tongue cancer in Sweden and Finland}

Mäkitie Antti ${ }^{1,2,3}$, Kamali Alexander ${ }^{6}$, Mroueh Rayan ${ }^{1}$, Lindford Andrew ${ }^{4}$, Koivunen Petri ${ }^{5}$, Autio Timo ${ }^{5}$, Lassus Patrik ${ }^{4}$, Halle Martin ${ }^{6}$, Bäck Leif ${ }^{1}$, Palmgren Björn ${ }^{3}$, and HammarstedtNordenvall Lalle ${ }^{3}$

${ }^{1}$ Department of Otorhinolaryngology - Head and Neck Surgery, University of Helsinki and Helsinki University Hospital, Helsinki, Finland

${ }^{2}$ Research Program in Systems Oncology, Faculty of Medicine, University of Helsinki, Helsinki, Finland

${ }^{3}$ Department of Clinical Science, Intervention and Technology, Department of Oto-RhinoLaryngology, Head and Neck Surgery, Karolinska Institutet, Karolinska University Hospital, Stockholm, Sweden

${ }^{4}$ Department of Plastic Surgery, University of Helsinki and Helsinki University Hospital, Helsinki, Finland

${ }^{5}$ Department of Otorhinolaryngology - Head and Neck Surgery, Oulu University Hospital, Oulu, Finland

${ }^{6}$ Department of Molecular Medicine and Surgery, Department of Reconstructive Plastic Surgery, Karolinska University Hospital, Stockholm, Sweden

Keywords: tongue cancer, reconstruction, outcome, recurrence

Running title: Differences in the treatment for tongue cancer

Financial support provided by: The Laryngeal Foundation, Karolinska Institutet and the ACTA Otolaryngologica Foundation in Sweden and the Helsinki University Hospital Research Fund and the Finska Läkaresällskapet in Finland.

No conflicts of interests were reported from any of the authors.

Correspondence to: Lalle Hammarstedt Nordenvall, MD, PhD

Department of Oto-Rhino-Laryngology, Head and Neck Surgery

Karolinska University Hospital,

17176 Stockholm, Sweden

lalle.hammarstedt-nordenvall@sll.se 
+4685177000 telephone

\section{Abstract}

Background and Aims: Stage II cancer of the tongue is mostly managed surgically both locally and regionally. However, indications for postoperative radiotherapy and reconstructive options vary between centers. This paper aims to describe differences in treatment in a geographically homogenous cohort.

Methods: A retrospective comparison was made between two cohorts of clinical T2N0 tongue cancer from Finland and Sweden. The Finnish cohort included 75 patients and the Swedish 54. All patients had curative intent of treatment and no previous head and neck cancer. Data analyzed consisted of pathological stage, size and thickness of tumor, frequency of reconstruction, radiotherapy delivered, and survival.

Results: The Finnish cohort included a higher proportion of patients managed with reconstructive surgery $(67 \%)$ than the Swedish cohort $(0 \%), \mathrm{p}<0.00001$. More patients were treated with postoperative radiotherapy (84\%) in the Swedish cohort than in the Finnish (54\%), $\mathrm{p}<0,0002$. The Finnish cohort had a higher level of survival and included more frequent downstaging (cTNM to pTNM).

Conclusions and significance: Our data indicate a major difference in the management of T2N0 oral tongue cancer. The optimal cut-off size and growth pattern of the tumor warranting reconstruction should be further evaluated in a prospective manner considering both survival and quality of life. 


\section{Introduction}

Oral tongue squamous cell carcinoma (OTSCC) is one of the most common head and neck cancers in the Nordic countries (Nordcan). There have been reports of an increasing incidence trend among young adults [1] and the current age-adjusted incidence (/100 000) in Sweden and Finland is 3,4 and 3,4 for women and 4,1 and 5,0 in men, respectively (Nordcan, 2016). The survival has not changed in Sweden during the last decades [2] but in Finland an improvement has been recently reported [3].

Early stage OTSCC is most often primarily managed surgically as a single-modality treatment or combined with postoperative (chemo)radiotherapy, and there are a great number of investigations addressing the treatment of the N0 neck [3-5]. An emerging alternative is the use of sentinel node biopsy and subsequent treatment of the neck based on the biopsy result [6-8]. Primary radiotherapy is used to a lesser extent, mainly in patients with significant comorbidities and advanced age and has been associated with a decreased overall and diseasespecific survival. However, these data are retrieved from register data $[9,10]$ and may be hindered by selection bias.

The recommendations for the postoperative treatment of stage II disease are mainly based on the histopathological assessment of the surgical specimen. The guidelines of the NCCN, the Swedish Head and Neck Cancer Register (SweHNR), and the Finnish Head and Neck Oncology Working Group recommend postoperative radiotherapy when adverse histopathological features are present, when the margin is close/positive or when the neck is 
positive. The SweHNR recommends PORT in T2 and larger lesions. These recommendations can, however, be broadly interpreted when for example patient-specific criteria such as comorbidities, preferences and age are addressed. Also, there is an obvious lack of randomized controlled trials regarding functional and quality of life (QoL) results of various reconstruction options in OTSCC patients. Therefore, scarce information exists regarding the indications for the use of free tissue transfers in the reconstruction of the surgical defect. The available data are most often single institution series and to our knowledge only one comparison study has been published with survival as the major outcome [11]. Therefore, the indications to reconstruct or not probably vary between centres and large variations may exist.

There are no clear guidelines regarding the reconstruction of a surgical tongue defect after cancer resection. We aimed to compare the OTSCC management protocols in Sweden and Finland due to the differing reconstructive approach for T2 OTSCC in these two Nordic countries. Therefore, we performed a descriptive analysis of the treatment of stage II OTSCC at the Karolinska University Hospital (Stockholm, Sweden) and at two university hospitals in Finland (Helsinki and Oulu). Since the guidelines for delivering postoperative radiotherapy differ we also aimed to investigate the clinical parameters governing the decision to reconstruct the lesion and to offer postoperative radiotherapy.

\section{Materials and methods}

All patients treated for clinically stage II (TNM7) OTSCC at the Karolinska University Hospital (Stockholm, Sweden; years 2008-2014 [5]) and at the Helsinki and Oulu University 
Hospitals (Finland; years 2004-2014 [3]) were included. Only patients treated with a curative intent for a first primary tumor in the head and neck region were included.

Clinical data on gender, age, tumor location, tumor thickness, histopathological assessment of primary tumor, macro- or microscopic tumor or isolated tumor cells on histopathological evaluation of the neck specimen, margin status, postoperative radiotherapy, presence of and type of reconstruction, local recurrence or isolated regional recurrence were recorded. Recurrence-free survival was analyzed as the time from diagnosis to recurrence. Overall survival was analyzed as time from diagnosis to death of any cause. Disease-specific survival was recorded as time from diagnosis to death caused by the disease. A second primary resulted in censoring.

Kaplan-Meier curves were constructed to describe the different survival trends and a log-rank test was performed to test for any difference(s). A stratified test for pT2N0 stages was also performed. Categorical data were analyzed using either the Chi-square test or Fishers exact test when appropriate. For continuous data, Student's t-test was used.

\section{Results}

At the Karolinska University Hospital, there were 54 patients diagnosed with a cT2NOM0 OTSCC and 75 patients at the Helsinki and Oulu University Hospitals during the study period

(Table 1). The median age at diagnosis did not differ between the two cohorts $(\mathrm{p}=0,3)$.

Differences in depth of invasion between the two cohorts did not reach statistical difference 
$(\mathrm{p}=0,8)$, but there was a significant difference in tumor diameter between the centers with a larger diameter in the Stockholm patients $(\mathrm{p}=0,0006)$.

Significantly more patients were treated with postoperative radiotherapy in the Stockholm cohort (84\%) than in the Finnish (54\%), p<0,0002. The patients in the Finnish series were reconstructed more often than in Stockholm, 67\% vs. $0 \%(\mathrm{p}<0,00001)$. The most frequently used flap type was the radial forearm free flap, which was used in $76 \%$ of the Finnish cases. Neck dissections were performed in patients treated in Stockholm equally as often as in the Finnish patients $(\mathrm{p}=0,888)$.

Regarding the pathological stage, a significantly higher proportion were down-staged after pathological evaluation to stage I in Finland than in Stockholm (39\% vs. 17\%; $\mathrm{p}=0,0073)$. Only two patients in the Finnish series and none in the Stockholm series were up-staged to T3. The main reason for up-staging were due to N-site positivity: $44 \%$ in Stockholm and 31 $\%$ in Finland $(\mathrm{p}=0,189)$. In Stockholm, $43 \%$ of the patients had the same pathological and clinical staging compared with $34 \%$ in Finland $(\mathrm{p}=0,30)$.

Five-year overall survival was 57\% in Stockholm and 73\% in Finland, disease-specific survival $65 \%$ and $89 \%$, and recurrence-free survival $62 \%$ and $77 \%$, respectively. Regarding the stratified test for pathological stage II patients the survival benefitted the Finnish cohort when disease-specific and overall survival were analyzed (Figures 1 and 2). When the data were analyzed for recurrence free-survival, no significant differences were seen between the curves (Figure 3). 


\section{Discussion}

This study reveals a difference in the treatment approach of early stage tongue cancer (cT2N0M0) within a geographically and culturally homogeneous population in the Nordic countries. The two major differences concerned the indications for postoperative radiotherapy and reconstruction of the surgical defect. Variations in the management protocol may have contributed to survival differences.

Administration of postoperative radiotherapy is based on pathological tumor stage, margins and the histopathological evaluation of the specimen. Also, performance status and comorbidities of the patient are considered in the decision making for postoperative radiotherapy. In this study, a significant difference was found in the use of postoperative radiotherapy between the two countries. However, one must consider that the differences in margin and histopathological assessment may explain these differences. For example, when the decision to administer radiotherapy is based on the pathological stage it is noteworthy that patients in the Finnish cohort were down-staged to a higher extent than those in the Stockholm cohort. However, this statement allows for broad interpretation and one should probably analyze differences prospectively between the different sites to assess eventual differences in the postoperative treatment of these patients. Subramaniam et al. reviewed the literature regarding the treatment of early stage tongue cancers and found that patients with multiple adverse pathological features were more likely to benefit from radiotherapy than patients with single adverse pathological features. However, this study was performed using the 7th TNM classification and one of the adverse features was tumor thickness [12]. The authors highlight the need for a re-evaluation of the topic using the latest TNM classification. 
The decision to reconstruct an oral tongue defect should be based on either the probability of achieving a greater tumor-free margin, hence reducing the risk of local recurrence, or providing a better QoL for the patient after the treatment. The decision to reconstruct the surgical defect or not was found to differ significantly between the two sites in the present study. There is clearly a more conservative approach toward microvascular reconstruction in Stockholm than in Finland. There seems to be a significant survival difference between the studied sites i.e. Finland having a much higher overall, recurrence-free and disease-specific survival. There is however, a slightly uneven distribution of pathological stages between these sites i.e. the Finnish series having overall significantly lower stages than the one from Stockholm. When stratifying for pT2N0 this survival difference remained except for recurrence-free survival. The basis for this finding is unclear but it might reflect a similar primary treatment effect in these cohorts, but a higher rate of successful salvage treatment in the Finnish one.

One of the difficulties in analyzing oral tongue tumors is the mixing of tumor sites in the analysis. Some authors report on tongue cancer specifically but there seems to be a mixture of stages included. The data are most often collected retrospectively and the histopathological aggressiveness is not considered. Also, regarding the surgical excision, there seems to be an incomplete definition of the resection type performed. An effort to harmonize the used technique and indications has been made by Ansarin et al. [13]. They described a definition for tongue surgery and the indications upon which the surgical technique should be used. This proposal may serve as a platform for more unified criteria to be used in the comparison between studies.

One of the indications to reconstruct a tongue defect with a free flap could be the intention to ensure adequate surgical margins whilst preserving deglutition function. The current 
oncological literature is scarce regarding the comparison between outcome after oral tongue reconstruction and non-reconstructive surgery. However, Lu et al. reported on the outcome difference between these approaches in a series of 347 OTSCC patients [14]. Single institutional data were grouped based on reconstructive approach. They found an increased pathological free margin and a relative lower recurrence and good survival rates in those patients who were reconstructed in comparison to those who were not. Although the data are retrospective and selection bias cannot be ruled out, this is an important paper highlighting the need for further investigations in this area. Also, the type of surgical resection included in the analysis needs to be defined more thoroughly.

There are few studies addressing the possible benefit of microvascular reconstruction after partial tongue resection. A prospective study from 1998 showed equal or better function in the primary closure group than in the reconstructed group in comparable locus of resection [15]. This study is however 20 years old and the patient inclusion is even older and there has been a great improvement in the available free-tissue transfer techniques since then. Ji et al. also performed a retrospective analysis between reconstruction and non-reconstruction in a series of 47 OTSCC patients evaluating speech, swallowing and tongue mobility 2-3 years after surgery [11]. However, their series included patients from partial to total glossectomies. They found a significantly better functional outcome for the non-reconstructed patients. These results are in line with data published from some other centers investigating the role of oral and oropharyngeal reconstruction $[15,16]$. When the resection is larger the literature is more supportive towards reconstruction. Ji et al. reported in a series of patients with hemiglossectomy or larger resections, a better tongue mobility, articulation, verbal diadochokinesis and speech intelligibility for those patients who were reconstructed in comparison to those who were not [11]. Canis et al. also found a superior QoL for patients with a T3 tongue cancer treated with reconstruction in comparison to those who were not 
reconstructed [17]. Both those studies are however retrospective in nature and may be exposed to confounding factors. According to Sun et al. the tumor site and size and thus, also the extent of tongue resection may be the key factors in determining the postoperative articulation intelligibility in glossectomy patients [18]. Despite the fact that QoL measurements include important parameters in head and neck oncology, there is still a remarkable lack of consistency and uniformity of reporting of functional outcomes in the literature [19]. This has been highlighted by Kansey et al. who described considerable variation in the need for reconstruction and in the appropriate reconstructive techniques among head and neck surgeons [20].

The retrospectively collected data in this study involves an inherent risk of bias. However, the study is descriptive and not analytic and aims to describe the present differences in treatment between two geographically and culturally homogenous areas. There is an indisputable difference in the reconstructive protocol between these centers. This difference should be further evaluated in a prospective setting to assess what stages and pattern of tumor growth warrant reconstruction. More importantly, the differences should be evaluated based on disease outcome and QoL measures.

\section{REFERENCES}

1. Annertz K, Anderson H, Palmer K, et al. The increase in incidence of cancer of the tongue in the Nordic countries continues into the twenty-first century. Acta otolaryngologica. 2012;132(5):552-7. 
patients with tonsillar, base of tongue and tongue cancer in Sweden. Oral Oncology. 2011;47(7):636-41.

3. Mroueh R, Haapaniemi A, Grenman R, et al. Improved outcomes with oral tongue squamous cell carcinoma in Finland. Head \& Neck. 2017;39(7):1306-12.

4. D'Cruz AK, Vaish R, Kapre N, et al. Elective versus Therapeutic Neck Dissection in Node-Negative Oral Cancer. The New England Journal of Medicine. 2015;373(6):521-9.

5. Kamali A, Gahm C, Palmgren B, et al. Regional recurrence in early stage I-II oral tongue cancer: a single institutional study and review of the literature. Acta Otolaryngologica. 2017;137(7):755-61.

6. Civantos FJ, Zitsch RP, Schuller DE, et al. Sentinel lymph node biopsy accurately stages the regional lymph nodes for T1-T2 oral squamous cell carcinomas: results of a prospective multi-institutional trial. Journal of clinical oncology : official journal of the American Society of Clinical Oncology. 2010;28(8):1395-400.

7. Agrawal A, Civantos FJ, Brumund KT, et al. [(99m)Tc]Tilmanocept Accurately

Detects Sentinel Lymph Nodes and Predicts Node Pathology Status in Patients with Oral Squamous Cell Carcinoma of the Head and Neck: Results of a Phase III Multi-institutional Trial. Annals of Surgical Oncology. 2015;22(11):3708-15.

8. Schilling C, Stoeckli SJ, Haerle SK, et al. Sentinel European Node Trial (SENT): 3-year results of sentinel node biopsy in oral cancer. European Journal of Cancer. 2015;51(18):2777-84.

9. Sowder JC, Cannon RB, Buchmann LO, et al. Treatment-related determinants of survival in early-stage (T1-2N0M0) oral cavity cancer: A population-based study. Head \& Neck. 2017;39(5):876-80. 
10. Gogarty DS, Lennon P, Deady S, et al. Variation in treatment and outcome in the early stage oral cavity squamous cell carcinoma. European Archives of Oto-RhinoLaryngology : official journal of the European Federation of Oto-Rhino-Laryngological Societies. 2017;274(2):953-60.

11.

Ji YB, Cho YH, Song CM, et al. Long-term functional outcomes after resection of tongue cancer: determining the optimal reconstruction method. European Archives of Otorhino-laryngology : official journal of the European Federation of Oto-Rhino-Laryngological Societies. 2017;274(10):3751-6.

12. Subramaniam N, Balasubramanian D, Murthy S, et al. Adverse pathologic features in early oral squamous cell carcinoma and the role of postoperative radiotherapy-a review. Oral surgery, oral medicine, oral pathology and oral radiology. 2017;124(1):24-31. 13. Ansarin M, Bruschini R, Navach V, et al. Classification of GLOSSECTOMIES Proposal for tongue cancer resections. Head \& neck. 2019;41(3):821-7.

14. Lu CC, Tsou YA, Hua $\mathrm{CH}$, et al. Free flap reconstruction for early stage tongue squamous cell carcinoma: surgical margin and recurrence. Acta oto-laryngologica. 2018;138(10):945-50.

15. McConnel FM, Pauloski BR, Logemann JA, et al. Functional results of primary closure vs flaps in oropharyngeal reconstruction: a prospective study of speech and swallowing. Archives of otolaryngology--head \& neck surgery. 1998;124(6):625-30. 16. Lee DY, Ryu YJ, Hah JH, et al. Long-term subjective tongue function after partial glossectomy. Journal of oral rehabilitation. 2014;41(10):754-8.

17. Canis M, Weiss BG, Ihler F, et al. Quality of life in patients after resection of pT3 lateral tongue carcinoma: Microvascular reconstruction versus primary closure. Head \& neck. 2016;38(1):89-94 
18. Sun J, Weng Y, Li J, et al. Analysis of determinants on speech function after glossectomy. Journal of oral and maxillofacial surgery : official journal of the American Association of Oral and Maxillofacial Surgeons. 2007;65(10):1944-50.

19. Ojo B, Genden EM, Teng MS, et al. A systematic review of head and neck cancer quality of life assessment instruments. Oral oncology. 2012;48(10):923-37.

20. Kansy K, Mueller AA, Mucke T, et al. A worldwide comparison of the management of T1 and T2 anterior floor of the mouth and tongue squamous cell carcinoma Extent of surgical resection and reconstructive measures. Journal of cranio-maxillo-facial surgery : official publication of the European Association for Cranio-Maxillo-Facial Surgery. 2017;45(12):2097-104. 


\begin{tabular}{|c|c|c|c|}
\hline & Swedish Cohort & Finnish Cohort & \\
\hline Patient characteristics & & & $p$-value \\
\hline Number & 54 & 75 & \\
\hline $\begin{array}{l}\text { Age-median and range } \\
(\%)\end{array}$ & $65(24-97)$ & $63(22-89)$ & $p=0,3$ \\
\hline $\begin{array}{l}\text { Gender male/female } \\
(\%)\end{array}$ & $22 / 32(41 / 59)$ & $36 / 39(48 / 52)$ & $p=0,4$ \\
\hline \multicolumn{4}{|l|}{ Tumor characteristics } \\
\hline $\begin{array}{l}\text { Diameter-median and } \\
\text { range }\end{array}$ & $26(12-40)$ & $21(3,5-49)$ & $p=0,0006$ \\
\hline $\begin{array}{l}\text { Invasion-median and } \\
\text { range }\end{array}$ & $9(2-19)$ & $8,1(1,5-30)$ & $p=0,8$ \\
\hline $\begin{array}{l}\text { Differentiation } \\
\text { low/mediate/high. (\%) }\end{array}$ & $13 / 27 / 14(24 / 50 / 26)$ & $22 / 36 / 9(33 / 54 / 13)$ & $p=0,189$ \\
\hline CTNM -T2NOM 0 & 54 & 75 & \\
\hline Cstage- stage 2 & 54 & 75 & \\
\hline \multicolumn{4}{|l|}{ pTNM } \\
\hline \multicolumn{4}{|l|}{ pstage } \\
\hline $\begin{array}{l}\text { pstage-1 } \\
\text { pstage-2 } \\
\text { pstage-3 } \\
\text { pstage-4 }\end{array}$ & $\begin{array}{l}8(17 \%) \\
23(48 \%) \\
7(15 \%) \\
10(21 \%)\end{array}$ & $\begin{array}{l}29(39 \%) \\
25(34 \%) \\
11(15 \%) \\
9(12 \%)\end{array}$ & $\begin{array}{l}p=0,0512 \\
p=0,30\end{array}$ \\
\hline \multicolumn{4}{|l|}{ pTstage } \\
\hline pT1 & 12 & 38 & $p=0,0073$ \\
\hline pT2 & 36 & 34 & $p=0,11$ \\
\hline \multicolumn{4}{|l|}{ pN-stage } \\
\hline pNO & $22(56 \%)$ & $42(69 \%)$ & \\
\hline pN1 & $8(21 \%)$ & $10(16 \%)$ & \\
\hline pN2 & $9(23 \%)$ & $9(15 \%)$ & $p=0,1889$ \\
\hline \multicolumn{4}{|l|}{ Treatment } \\
\hline Surgery-local (\%) & $49(90 \%)$ & $75(100 \%)$ & $p=0,0053$ \\
\hline tneckdissection (\%) & $39(80 \%)$ & $61(81 \%)$ & $p=0,888$ \\
\hline $\begin{array}{l}\text { Reconstruction of } \\
\text { primary site (\%) }\end{array}$ & $0(0 \%)$ & $50(67 \%)$ & $p \varangle 0,00001$ \\
\hline Partial glossectomy & $31(63 \%)$ & $39(52 \%)$ & $p=0,229$ \\
\hline Hemiglossectomy & $18(37 \%)$ & $34(45 \%)$ & $p=0,379$ \\
\hline Subtotal glossectomy & 0 & $2(3 \%)$ & $p=0,321$ \\
\hline \multicolumn{4}{|l|}{ Flaps } \\
\hline$-A L T$ & 0 & $8(16 \%)$ & NA \\
\hline -RFA & 0 & $38(76 \%)$ & NA \\
\hline -lateral arm & 0 & $2(4 \%)$ & NA \\
\hline -pectoral & 0 & $2(4 \%)$ & NA \\
\hline $\begin{array}{l}\text { Adjuvant radiotherapy } \\
\text { (\%) }\end{array}$ & $42(86 \%)$ & $41(54 \%)$ & $p=0,0002$ \\
\hline $\begin{array}{l}\text { Adjuvant } \\
\text { chemoradiation (\%) }\end{array}$ & $9(18 \%)$ & $7(9 \%)$ & $p=0,157$ \\
\hline
\end{tabular}




\begin{tabular}{|l|l|l|l|}
\hline Only radiotherapy & 5 & 0 & NA \\
\hline Only chemoradiation & 2 & 0 & NA \\
\hline
\end{tabular}




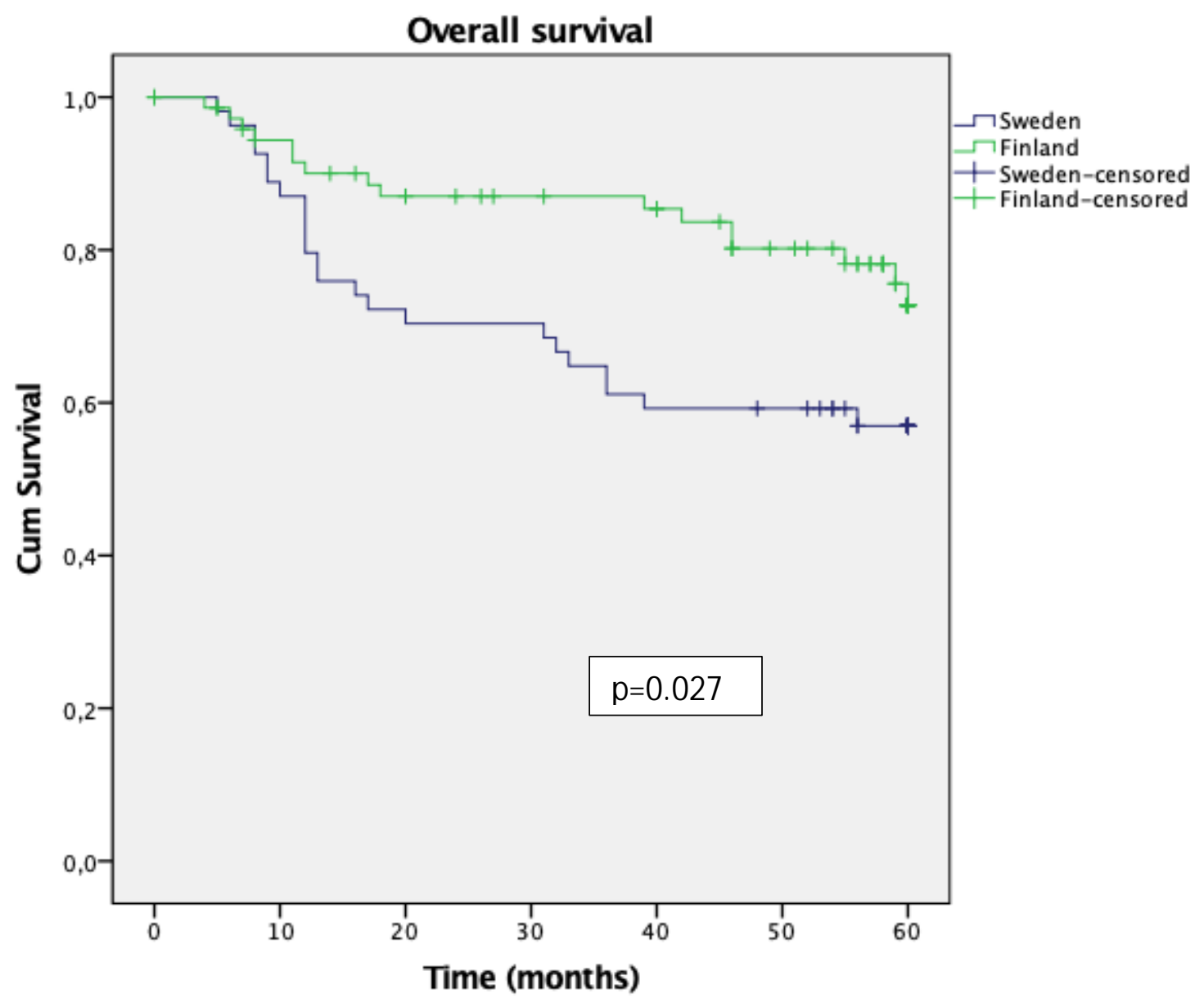




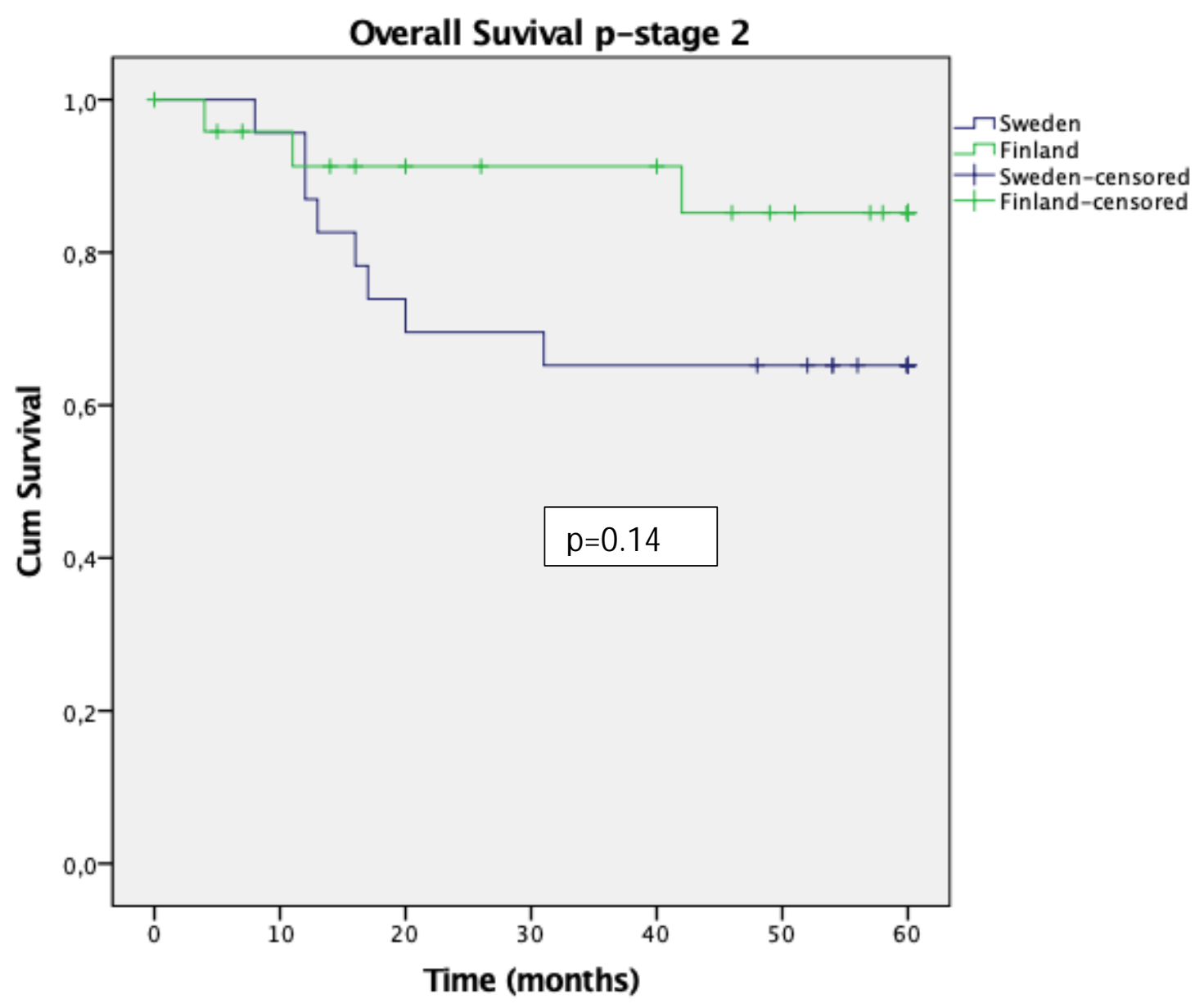




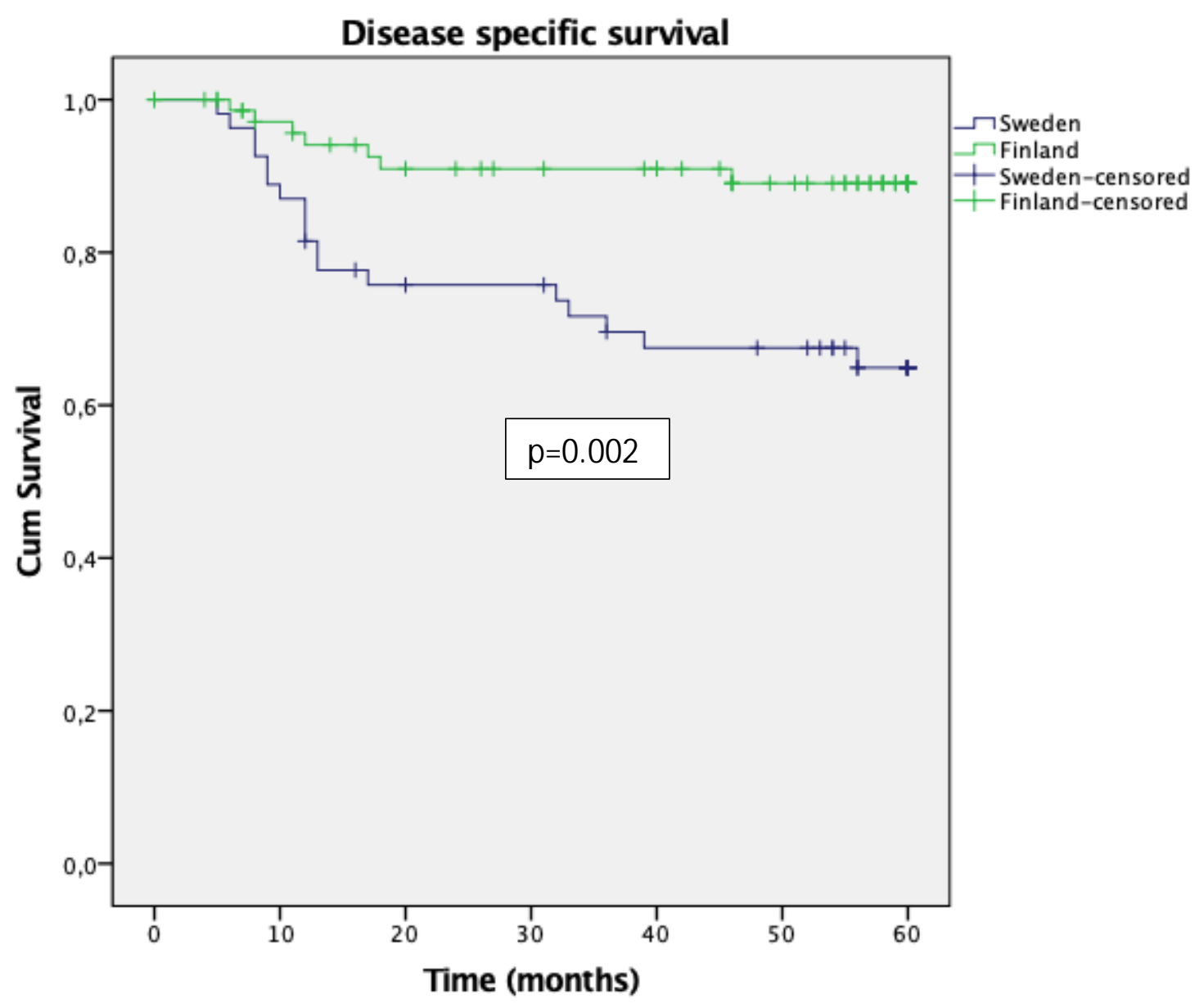




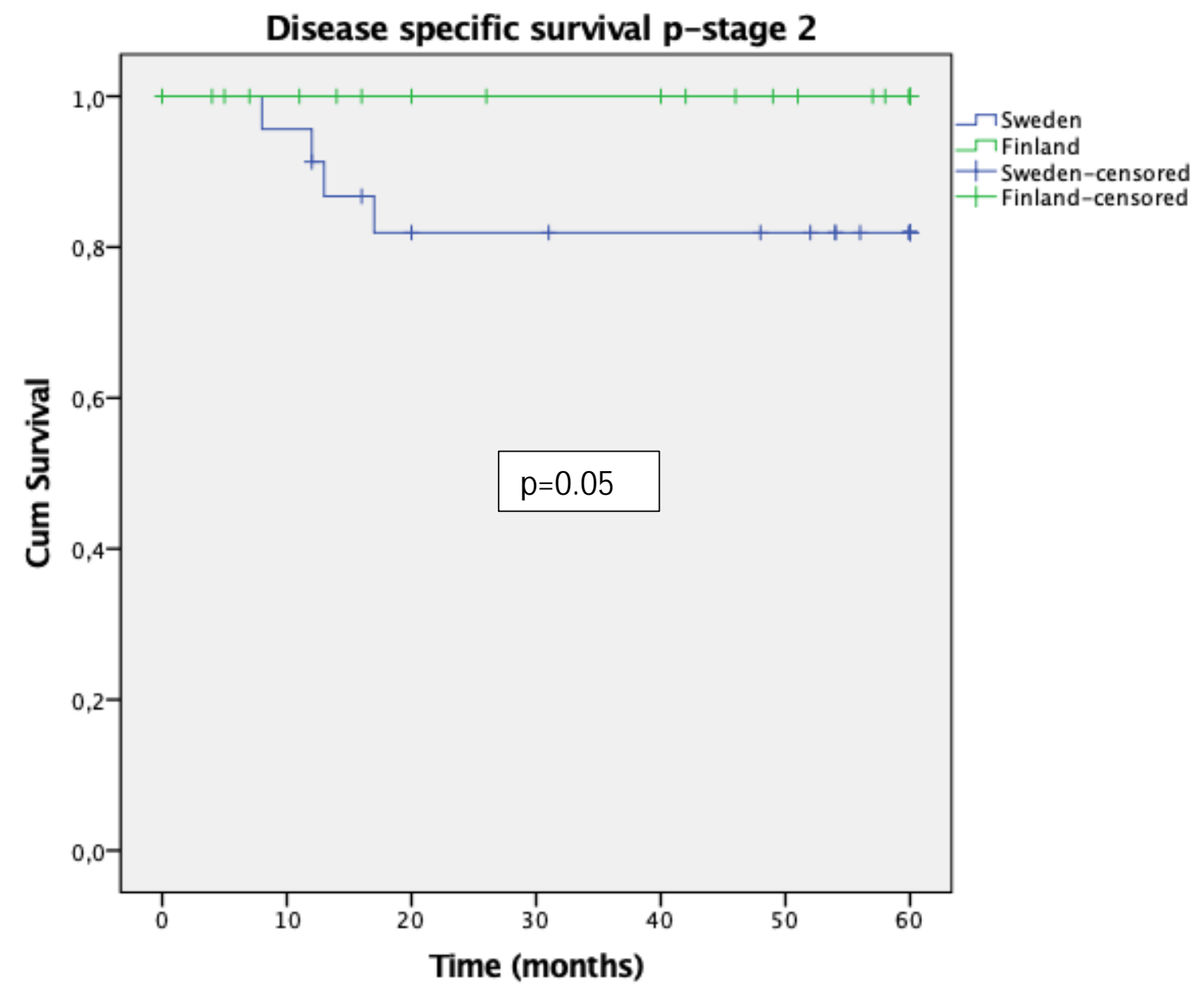




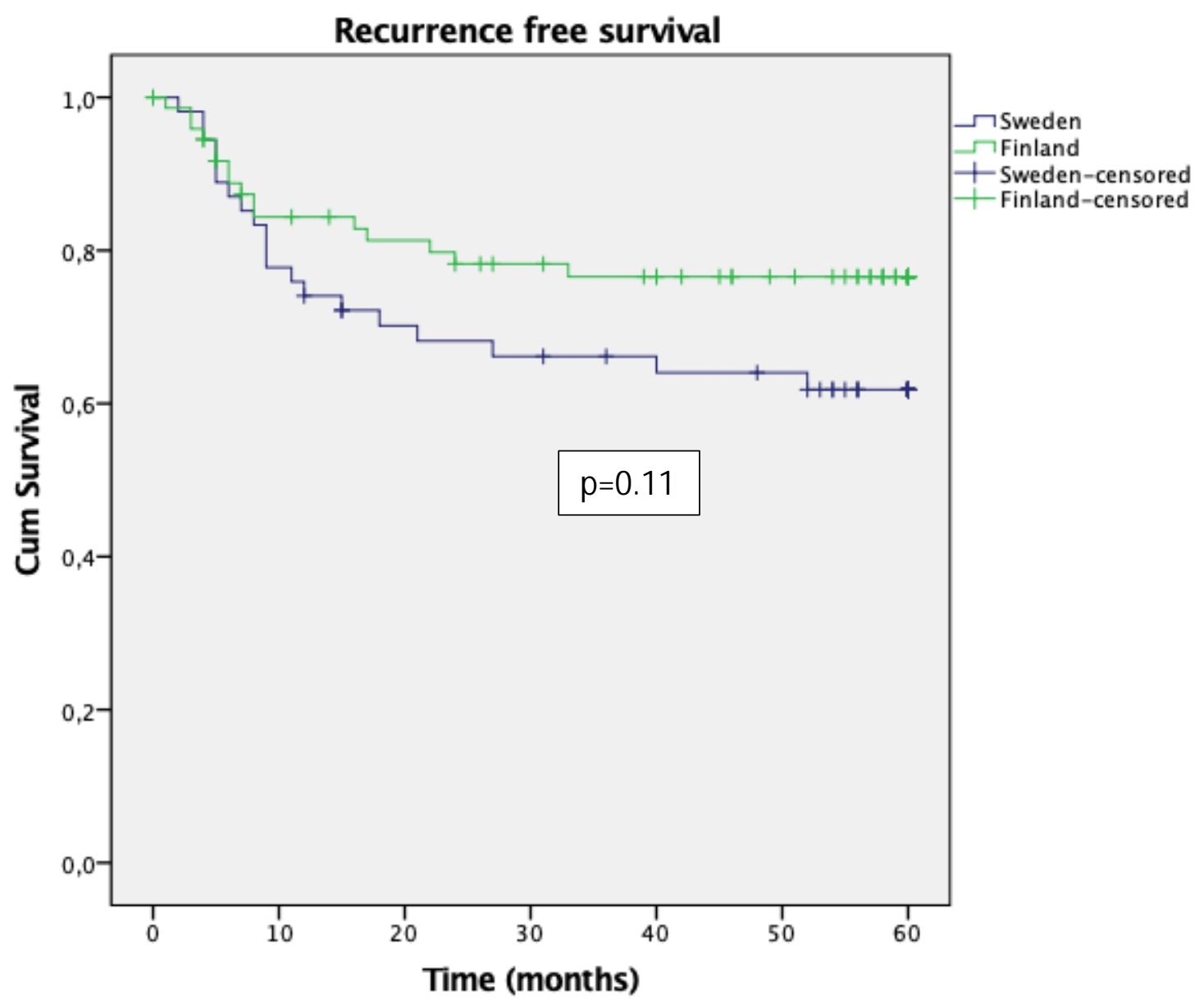




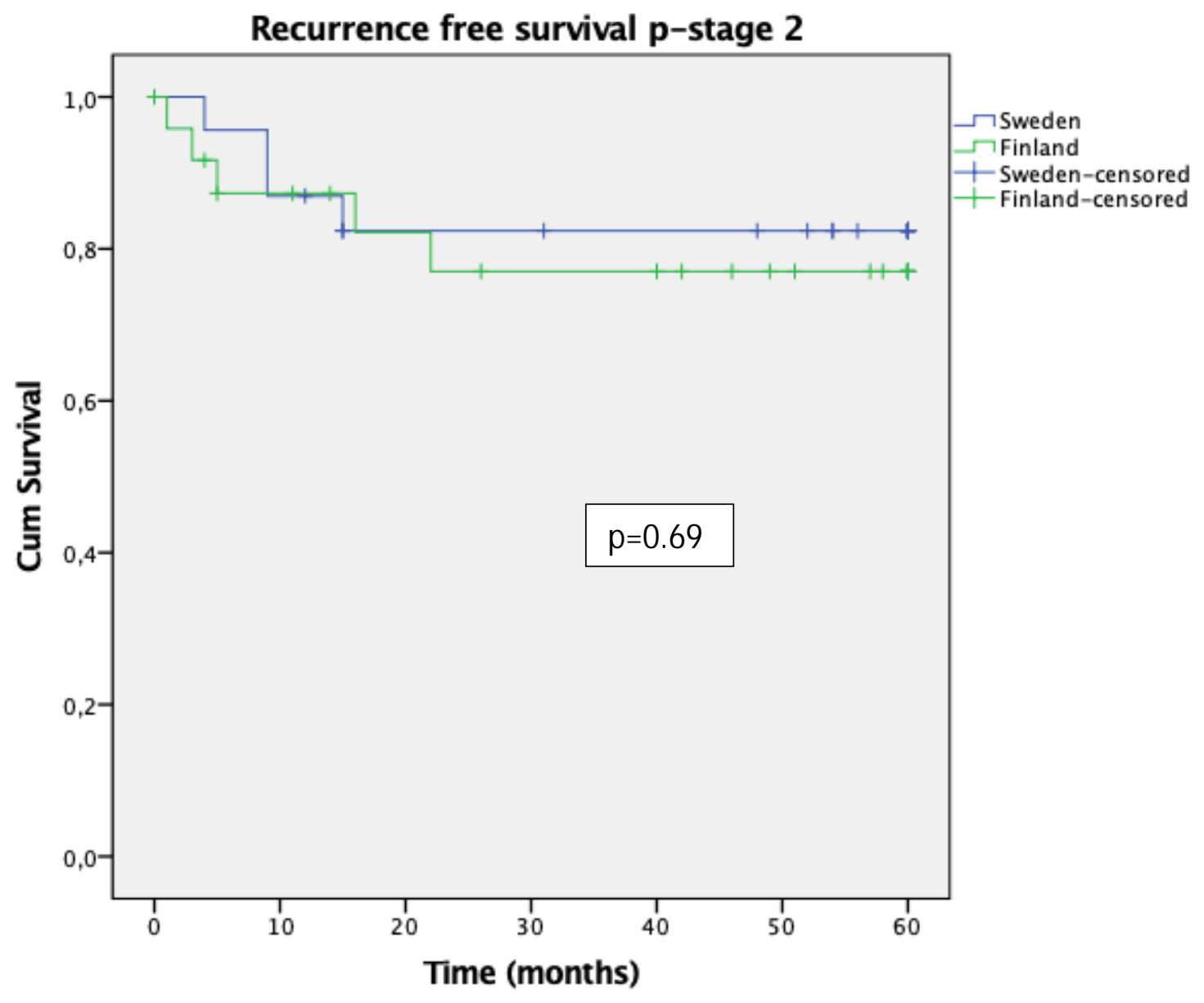

